\title{
TIME-RESOLVED IMAGING OF ELECTRON AND POSITRON BEAMS AT APS
}

\author{
A. H. Lumpkin and B. X. Yang \\ Advanced Photon Source, Argonne National Laboratory \\ 9700 South Cass Avenue, Argonne, Illinois 60439 LSA
}

CONF- $970503-62$

\section{Abstract}

Characterizations of stored electron beams and more recently positron beams circulating in the Advanced Photon Source (APS) 7-GeV storage ring have been done using optical synchrotron radiation (OSR) and $x$-ray synchrotron radiation (XSR) imaging techniques. Results include the measurement of the bunch length and horizontal beam size versus single-bunch current for both electron and positron beams, observations of multibunch bunch length effects near $100 \mathrm{~mA}$ stored beam current, and initial diagnostics of a coupled-bunch instability with 15-minute period. Both the Hamamatsu C5680 dual-sweep streak camera using OSR and a pinhole camera using XSR from a bending magnet source point were utilized. Proposed enhancements to the beamline will also be presented.

\section{INTRODLCTION}

The transition of the Advanced Photon Source (APS) 7-GeV storage ring from the commissioning phase with electrons to the operations phase with positrons [1] has been supported by the use of time-resolved imaging techniques based on synchrotron radiation. Characterizations of the stored beams' bunch length and transverse size have been done using both optical synchrotron radiation (OSR) and $\mathrm{x}$-ray synchrotion radiation (XSR) from a bending magnet (BM) source point. As part of the program to evaluate the impedance of the ring. single-bunch studies of these parameters as a function of single-bunch current have been performed. Bunch lengthening effects have been observed to be more significant than energy spread growth with an increase in current. These results have been compared to predictions of a potential well distortion (PWD) model and a microwave instability calculation, and the data are found to be more consistent with the PWD calculatio. 2]. Additionally, appar it coupled-bunch instabilities: . .e been diagnosed via he increase in observed horizontal beam size at this dispersive point in the lattice as well as a separ: a bunch length measurement. The techniques will be briefly desuribed followed by examples of results.

\section{EXPERIMENTAL BACKGROUND}

As described elsewhere [3], the APS storage ring diagnostics include a bending magnet source dedicated to beam characterizations. The source point at about the oneeighth length of the dipole also is at a dispersive point in the lattice which allows access to the energy spread of the beam. Both optical synchrotron radiation (OSR) and
XSR-based techniques are used. Initially, a split, watercooled pickoff mirror was used to direct the OSR out of a window and into an optical transport while allowing the high power, lower-divergence $x$-ray beam to pass on to an in-air pinhole aperture. At present the front-end vacuum transport line includes a mask that separates the BM radiation into three pieces. One part continues to an in-vacuum 4-jaw aperture that acts as the pinhole aperture, and a second part strikes a single mirror that is shadowed from $x$ rays by a horizontally on-axis, cylindrical rod.

The $x$-ray images are converted to visible light at a $\mathrm{CdWO}_{4}$ converter and viewed by a Questar telemicroscope with a charge-coupled-device (CCD) camera. The visible, OSR is transported out of the tunnel to an optics lab which has a C5680 dual-sweep streak camera for bunch length measurements.

\section{EXAMPLES OF EXPERIMENTAL RESULTS}

\section{I Transverse Beam Size}

Measurements of the transverse beam size have been done within machine studies periods and the routine observation plans for beam quality with 100-mA stored beam. The observed beam sizes have been consistent with the baseline design of $8.2 \mathrm{~nm}$-rad natural emittance and better than the $10 \%$ vertical coupling design in almost all instances of user runs. In Fig. 1 an example of the x-ray pinhole image in a recent run is shown. The values of the

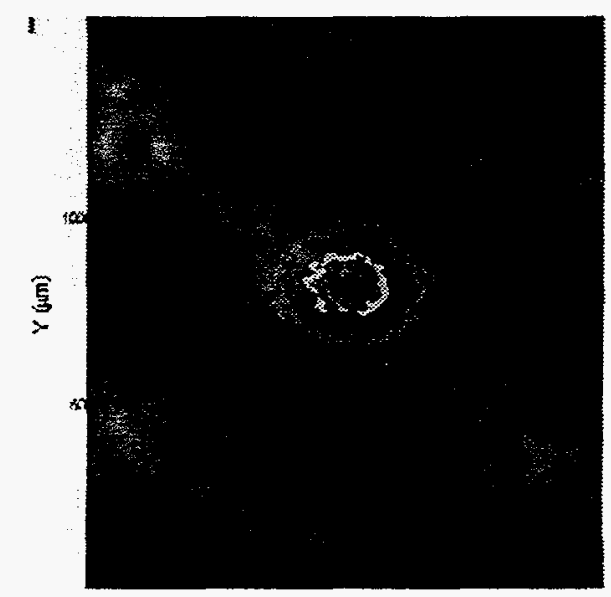

Figure 1: Example of an $x$-ray pinhole image of the APS stored positron beam at about $100 \mathrm{~mA}$. The horizontal $\left(\sigma_{x} \sim 160 \mu \mathrm{m}\right)$ and verrical sizes $\left(\sigma_{y} \sim 90 \mu \mathrm{m}\right)$ are consistent with the baseline design but with vertical coupling less than $10 \%$.

The submitted manuscript has been created by the Universiry of Chicago as Operator of Argonne National Laboratory ("Argonne") under Contrict No. W-31-109-ENG-38 with the U.S. Deparment of Energy. The U.S. Government retains for itselt, and others acting on its behalt, a paid-up, nonexclusive. irtevocable worldwide license in said aricle to reproduce. prepare derivative works. distribute copies to the public, and perform publicly and display publicly, by or on behulf of the Government. 


\section{DISCLAIMER}

This report was prepared as an account of work sponsored by an agency of the United States Government. Neither the United States Government nor any agency thereof, nor any of their employees, makes any warranty, express or implied, or assumes any legal liability or responsibility for the accuracy, completeness, or usefulness of any information, apparatus, product, or process disclosed, or represents that its use would not infringe privately owned rights. Reference herein to any specific commercial product, process, or service by trade name, trademark, manufacturer, or otherwise does not necessarily constitute or imply its endorsement, recommendation, or favoring by the United States Government or any agency thereof. The views and opinions of authors expressed herein do not necessarily state or reflect those of the United States Government or any agency thereof. 


\section{DISCLAMMER}

Portions of this document may be illegible in electronic image products. Images are produced from the best available original docoment. 
total observed size, $\sigma_{\mathrm{xT}} \approx 160 \mu \mathrm{m}$ and $\sigma_{\mathrm{yT}} \approx 90 \mu \mathrm{m}$, are representative of the runs in recent months with both the 6 +200 bunch pattern and the $6+17$ triplets bunch pattern. Using the calculated $\beta_{x}=1.8 \mathrm{~m}$ and $\beta_{y}=18.4 \mathrm{~m}$, the measured dispersion $\eta_{\mathrm{x}}=85 \mathrm{~mm}$, and the estimated system resolutions of $\sigma_{x \text { res }}=60 \mu \mathrm{m}$ and $\sigma_{\text {yres }}=40 \mu \mathrm{m}$, a vertical coupling of about $4-5 \%$ is indicated. These patterns involve a trade on beam lifetime and compatibility with time-resolved user experiments.

In the fall of 1996, a different fill pattern was employed involving four trains of 12-bunches positioned at $90^{\circ}$ intervals around the ring. Each 12-bunch set was initially filled to $25 \mathrm{~mA}(\sim 2 \mathrm{~mA} /$ bunch $)$ average current. An unanticipated phenomenon occurred as exhibited by the significant increase in horizontal beam size. This was observed to have about a $15-\mathrm{min}$ period which persisted from $100 \mathrm{~mA}$ down to $60 \mathrm{~mA}$ early on a user run. Figure 2 shows the plot of horizontal size (FWHM) measured in pixels or channels of the video processing system. The normal value of -12 ch at $28 \mu \mathrm{m} / \mathrm{ch}$ corresponds to $\Delta \mathrm{x}=$ $336 \mu \mathrm{m}(\mathrm{FWHM})$ or $143 \mu \mathrm{m}(\sigma)$. The maximum of the size increase is almost twice as large as the baseline value. There was very little change in the vertical size. Since this source point is at a dispersive point in the lattice, a longitudinal instability effect was likely involved. The 15-min time scales were traced to oscillations of the rf cavity temperatures as the cooling water regulator valves opened and closed. As shown in Fig. 3, the S37 cavity temperatures display a less than 1-degree oscillation at the $15 \mathrm{~min}$ period. EPICS [4] allowed the tracking of the temperature process variable and the beam size process variable together. Since it was determined that the instability was worse at the cavity temperature minima, the set points for the rf cavity temperatures were raised, and the instability was suppressed. Further details on the if side are given by Harkay et al. [5].

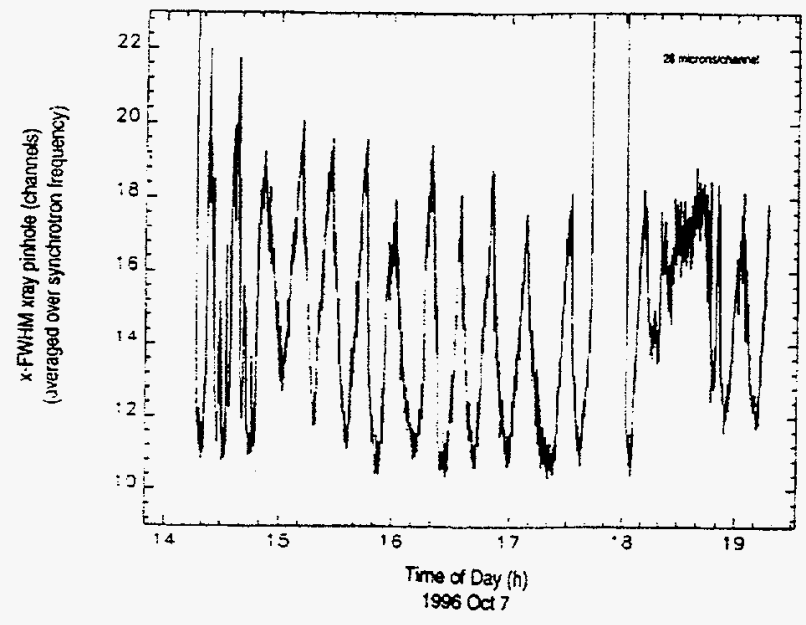

Figure 2: Plot of the variation of the horizontal beam size (positrons) in channels (FWHM) versus time. A significant size growth is seen with a 15 -min period.

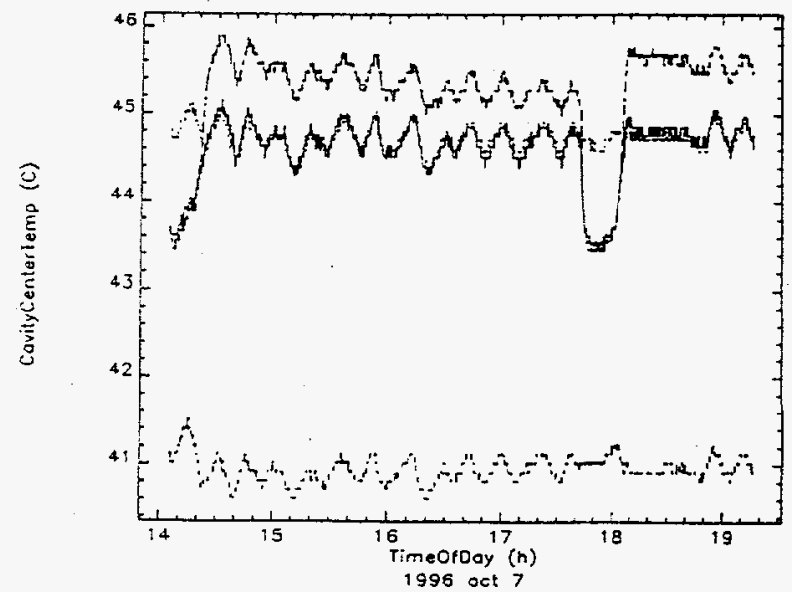

Figure 3: Plot of the variation of the sector $37 \mathrm{rf}$ cavity temperature probe versus the same time as Fig. 2. The maxima in Fig. 2 align with the temperature minima for this set of cavities.

\subsection{Longitudinal Phase-Space Effects}

Previously, we reported measurements in singlebunch studies which involved lower if gap voltages and synchrotron frequencies [2]. A more extensive set of data were obtained in June 1996 with electrons that addressed both the observed horizontal beam size at the energy dispersive source point and the longitudinal bunch profile and duration. Figure 4 shows the beam size and bunch length versus single-bunch currents up to $18 \mathrm{~mA}$ on the upper half and lower half of the figure, respectively. The bunch length (triangles) clearly more than doubles and could be fit to the $I^{1 / 3}$ dependence. The horizontal beam size (circles), and hence the effective energy spread, does not show any significant increase up to $12 \mathrm{~mA}$, but there does seem to be some increase after that. These combined effects are consistent with calculations done using the potential well distortion (PWD) model rather than the predicted $21 / 2$ times energy spread increase from a microwave instabilitybased scenario [2]. It is also noted that the peak current attained was about $400 \mathrm{~A}$. Subsequently, bunch length measurements have been taken for stored positron beams up to $7.5 \mathrm{~mA}$. These data show a similar bunch current dependency as the electron beam data.

An additional phenomena was observed in multibunch mode in dual-sweep streak images taken near $100 \mathrm{~mA}$, the baseline stored beam current. At $101 \mathrm{~mA}$ the observed bunch lengths were almost 190 ps (FWHM) (Fig. 5) versus 70 ps (FWHM) shortly thereafter when the current was 99 $\mathrm{mA}$. This appears to be an alternative measurement of the signature of a coupled-bunch instability which was related to the rf cavity higher-order modes (HOMs) at that time. These particular data were taken during our first attainment of $100 \mathrm{~mA}$ on January 12,1996 and are likely related to the complementary type measurement shown in Fig. 2. 


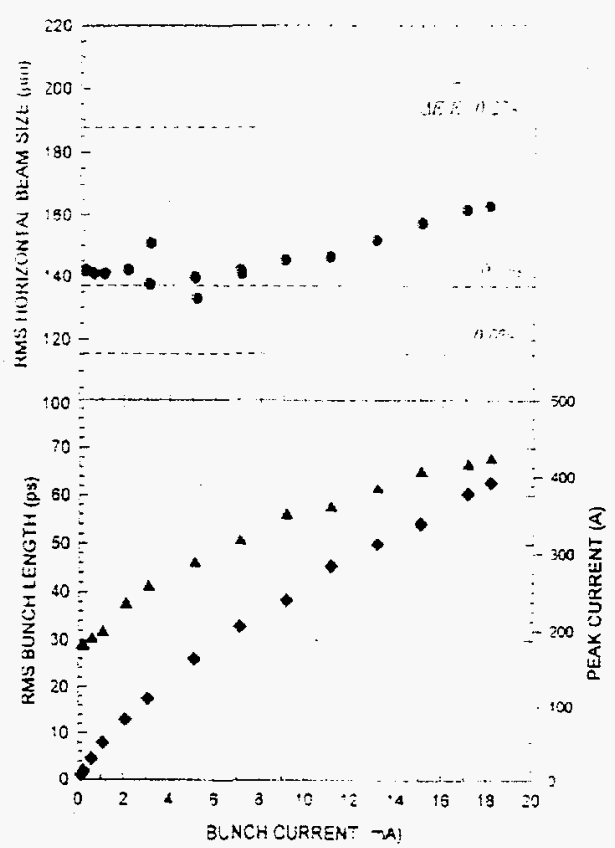

Figure 4: Plot of the rms beam size and bunch length versus single bunch current (electrons) taken on June 30, 1996. Significant bunch lengthening is observed but without comparable energy spread growth. The dashed lines in the upper figure show the 0.0,0.1, and $0.2 \% \mathrm{cE} / \mathrm{E}$ calculated sizes. In the lower half of the figure, the trianges (left-hand axis) are bunch length values and the diamonds are the peak current values (right-hand axis).

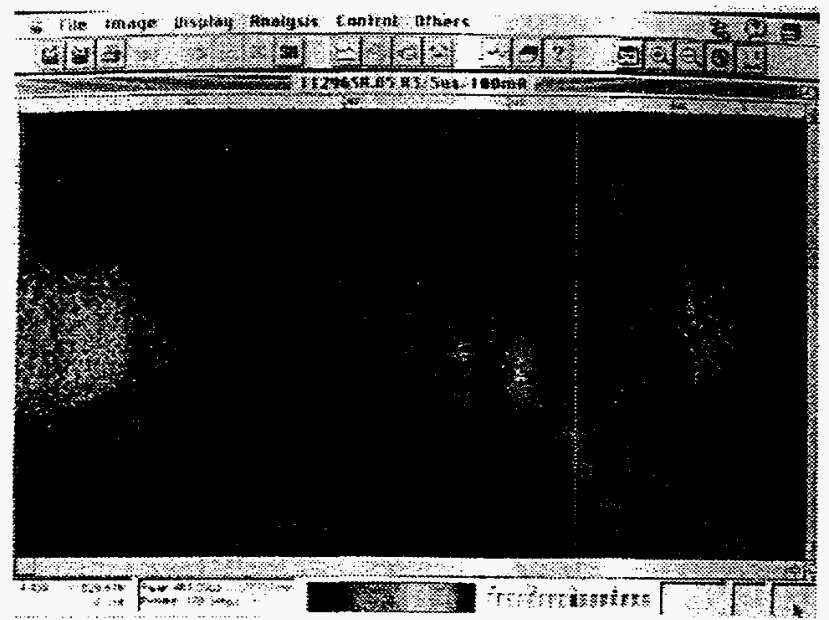

Figure 5: Dual sweep streak images of the stored beam at $101 \mathrm{~mA}$. The bunch length changed from $170 \mathrm{ps}$ (FWHM) in this image to $70 \mathrm{ps}$ (FWHM) at $\mathrm{I}_{\mathrm{B}}=99$ $\mathrm{mA}$. The vertical scale is $800 \mathrm{ps}$ and the horizontal scale is $5 \mu$ s The vertical white lines denore thic regionof-interest for the time protile of the multi-bunch train. Individual micropulses are not seen on this time wale. The next pass of the pulse train is seen at the left of the image where the vertical crotile is displayed.

\section{SUMMARY}

In summary, we have used the time-resolved imaging techniques to monitor the APS storage-ring beams under a variety of conditions. The transverse beam size measurements are now on-line and archived in data loggers with EPICS tools. Additionally, longitudinal effects as revealed in energy spread or bunch length have also been tracked. These techniques are now being complemented by a diagnostics undulator which can directly address beam divergence [6].

\section{ACKNOWLEDGMENTS}

The authors acknowledge the APS/ASD support of John Galayda and Glenn Decker as well as the assistance in the main control room of Louis Emery, Mike Borland, and Nick Sereno. APS work was supported by the U.S. Department of Energy, Office of Basic Energy Sciences, under Contract No. W-31-109-ENG-38.

\section{REFERENCES}

[1] Glenn Decker, "Performance of the Advanced Photon Source," these proceedings.

[2] A. H. Lumpkin, B. X. Yang, and Y. C. Chae, "Observations of Bunch Lengthening Effects in the APS 7-GeV Storage Ring," Nucl. Instrum. Methods, in press.

[3] B. X. Yang and A. H. Lumpkin, "Particle Beam Profiling Techniques on the APS Storage Ring: Commissioning Results," AIP Conf. Proc. 390, p. 491 (1997).

[4] EPICS or Experimental Physics and Industrial Control System, e.g., K. Sidorowicz and W. P. McDowell, "The APS Control System Network," Proceedings of ICALEPCS, Oct. 30-Nov. 3, 1995, Chicago, IL, p. 68.

[5] K. Harkay et al., "Compensation of Longitudinal Coupled-Bunch Instability in the APS Storage Ring," these proceedings.

[6] B. X. Yang et al., "Status of the APS Diagnostics Undulator Beamline," these proceedings. 